\title{
CHARACTERIZING DATA BASE DEPENDENCIES
}

\author{
J.A. Makowsky* \\ Faculty of Computer Science \\ Technion - Israel Institute of Technology \\ Haifa, Israel
}

ABSTRACT: We characterize the class of Full Implicational Dependencies introduced by Fagin as the maximal class of dependencies which are safe, admit finite models in a strong sense and admit Armstrong Relations. We also give other more modeltheoretic characterizations of Full and Embedded Inplicational Dependencies.

\section{INTRODUCTION}

Data base dependencies are first order formlae that can be specified to hold for a given relational data base. They form a basis for organizing semantic knowledge about data bases. They are also invaluable for structuring a data base so as to avola various problems, or anomalies, having to do with inserting and deleting data (cf. [U]). In recent years several proposals have been made for reasonable sets of dependencies $[A B U, B V, D F 77, G J, N, P, P P, P Y, R 78, S U, S W, T K Y 78$ and U] and attempts have been made to unify all these notions. Two classes of particular interest emerged in the 1iterature: The set of Embedded Implicational Dependencies (EID) as the largest class so far considered [F80, PY, CLM] including the Template Dependencies [US] and the Fu11 Implicational Dependencies (FID) [F80].

In [CLM] some evidence is presented that the EID form too large a class: the inference problem for EID is undecidable and there are ETDs which admit only infinite relations; in contrast this is not so for the FIDs. The main result of this paper is a characterization of the FIDs in terms of properties which emerge from data base theory, $i . e$. safety, securability and admitting Armstrong relations. The idea behind such characterizations is to delimit the use of full first order logic in data base theory and to show that these 1imitations are inherent to the subject. Previously [CLM] mode1 theoretic characterizations of both the ETDs and FIDs have been given, but we feel

* During part of the work supported by Swiss National Science Foundation Grant No. $82.820 \cdot 0.80$. 
that the characterizing properties had too much of an algebraic flavour and too little to do with data base theory.

The properties involved here are well established: Domain invariance or safety stems from the fact that we do not consider general models, but rather relations as such. So, if we want to use model theory, we have to justify that our dependencies only speak about the relation. The existence of Armstrongrelations can be considered fundamental to data base theory, especially if they are finite. They allow the designer of the data base model to gain a thorough overview of what he already assumes to be true. The notion of securability is new to data base theory, though it seems implicite in many considerations: it is a property that not only guarantees the existence of finite relations satisfying a given finite set of dependencies, but it guarantees it continuously: the property holds or fails in a given set of data provided some finite configuration of data is present. The notion was introduced by Tharp in a different context [Th] and further studied in [Ma]. Now the FIDs satisfy all of these demands and the main theorem tells us that no extension of the FIDs will do so. Extending the FIDs may still be reasonable, but we have to pay a price: Undecidability of the satisfaction and consequence relations is likely by [CLM] and violation of one of the above demands sure.

The $\mathrm{plan}$ of the paper is as follows: In Section 2 we review and present new model theoretic characterizations of the EIDs and FIDs and present a new characterization of safe dependencies. Other such characterizations of safety may be found in [PY and Co]. In Section 3 we present the concept of securability and in Section 4 we discuss sets of dependencies having the Armstrong property. A11 this is collected in Section 5 to prove our main result. Detailed proofs will appear elsewhere.

\section{SOME MODEL THEORY}

Let $\underline{R}$ be a relation symbol of arity $n$. A model $M=(D, R)$ for $\underline{R}$ consists of a set $D$ (the domain) together with a relation $R \in D^{n}$. Throughout, we assume that first order formulae have just one non-logical symbol $\underline{R}$ and may contain equality. An atomic formula is a formula of the form $\underline{R}\left(x_{i_{1}}, \ldots, x_{i_{n}}\right)$ or $x_{i}=x_{j}$ where the 
$x_{i}$ 's are variables. An (untyped or general) embedded implicational dependency, or EID for short, is a sentence of the form

$$
\forall x_{1}, \ldots, x_{p}\left(\left(A_{1} \wedge \ldots \wedge A_{q}\right) \rightarrow \exists y_{1}, \ldots, y_{x}\left(B_{1} \wedge \ldots \wedge B_{s}\right)\right)
$$

where each $A_{i}, B_{j}$ is an atomic formula. We will assume that each $x_{i}$ appears in at least one of the $A_{j}{ }^{\prime} s$. W.1.o.g., it may be assumed that no $A_{j}$ is an equality and equaligy in the $B_{i}^{\prime} s$ only uses $x_{i}$ 's. An EID is said to be full if it has no existential quantifier. We abbreviate full implicational dependencies by FID.

An EID (FID) is said to be typed if no variable occurs in two different columns (places) in $\underline{R}$, and equality occurs only between variables appearing in the same column. Models for types EIDs could be replaced by many-sorted models $M=\left(D_{1}, \ldots, D_{n} ; R\right)$ with $R \subset D_{1} \times D_{2} \times \ldots \times D_{n}$. A model is trivial if $|D|=1$ and $R=D^{n}$ (in the typed case this amounts to each $D_{i}$ being a singleton $\left\{d_{i}\right\}$ and $R=\left\{\left(d_{1}, d_{2}, \ldots, d_{n}\right)\right\}$. Given a set of formulae $\Sigma$ and a formula $\varphi$, we say $\Sigma=\varphi$ ( $\varphi$ is a consequence of $\Sigma$ ) if $\varphi$ holds in all (finite and infinite) models of $\Sigma$. We write $\Sigma=f \varphi$ if $\varphi$ holds in all finite models of $\Sigma$.

\subsection{Domain Invariance (Safety, Permissibility)}

A first order formula $\varphi$ is said to be Domain independent [F80] provided $\varphi$ holds in a model $(D, R)$ iff it holds in a model $\left(D^{\prime}, R^{\prime}\right)$ with $R=R^{t}$. Domain independence is called safety in $[U, p .113]$ and safe formulae are called permissible in [Co]. Note that "domain-independent" is used in [P4] for what we call "typed".

In [CLM] we defined a dependency to be a domain independent formula that holds in the trivial structure.

Note that the FIDs and EIDs are indeed dependencies.

Let $\varphi$ be a first order formula in prenex normal form with matrix in disjunctive normal form $\quad \underset{j}{\hat{j}} \varphi_{i j}$.

We define a variable $x$ to be constrained following [Co]:

(i) A direct constraint on $x$ is an atomic formula $R(\ldots . .$.$) in which x$ occurs.

(ii) An indirect constraint on $x$ is a formula $x=y$ where $y$ is directly constrained. 
(iii) If $x$ is existentially quantified in $\varphi$ then $x$ is constrained iff in every disjunct $A \varphi_{i j}$ in which $x$ occurs some $\varphi_{i j}$ is a direct or indirect constraint on $x$.

(iv) If $x$ is universaly quantified in $\varphi$ then $x$ is constrained iff in some disjunct $\wedge \varphi_{i j}$ in which $x$ occurs, every $\varphi_{i j}$ in which $x$ occurs is the negation of a direct or indirect constraint on $x$.

Now let $F_{i}(z)$ be the formula $\exists x_{1} x_{2} \ldots x_{i-1} x_{i+1} \ldots x_{n} \underline{R}\left(x_{1}, \ldots x_{i-1}, z, x_{i+1} \ldots x_{n}\right)$ and $F(z)$ be $v_{i}^{n_{i}} F_{i}(z)$. If $\varphi$ is a first order formula we denote by $\varphi F$ the formula which is obtained from $\varphi$ by inductively replacing each bound variable occurrence $\exists x$ by $\exists x(F(x) \wedge \ldots)$ and $\forall x$ by $\forall x(F(x) \rightarrow \ldots)$.

The following is an easy observation (cf. [Co], [Va]).

Proposition 1: Let $\varphi$ be a first order formula which holds in the trivial model. Then the following are equivalent:

$$
\varphi \text { is a dependency; }
$$

(ii) $\varphi$ is equivalent to $\varphi F$;

(iii) $\varphi$ is equivalent to a fomula $\varphi_{0}$ in which each bound variable is constrained. This can be read as a characterization of safe formulae in syntactic terms. Since $\varphi$ may contain arbitrary complex "superfluous" parts, we cannot replace " $\varphi$ is equivalent to..." by " $\varphi$ is of the form...". Proposition 1 states precisely and improves the observation in [Co]. We predict that this will be a "folk theorem" in the sense of [Ha].

Proposition 1 is a special case of an easy theorem in model theory characterizing sentences preserved under relativizations. Its origin goes back to the 1950 period. A more refined version involving theories was proved using ultraproducts by Keisler [Ke] and [CK, problem 5,2.20]. Note that Vardi observed [Va] that dependencies are recursive enumerable but not recursive. See also section 6 .

\subsection{Mode1 Theoretic Characterizations of FID and EID}

In [CLM] several characterization theorems for FID and EID were exhibited. We restate these results here and add some new ones. Undefined model theoretic and algebraic 
concepts may be found in the standard text book [CK]. We denote by $\prod_{i \in I}$ and $\lim _{i \in I} M_{i}$ the product and direct limit (for homomorphisms) of a family $M_{i} i \in I$ of structures. We say that a dependency (formula) $\varphi$ is:

(i) a substructure dependency (formula) if $M \neq \varphi$ and $M_{0} \subset M$ is a substructure then $M_{0}=\varphi$.

(ii) a product dependency (formula) if $M_{i} \neq \varphi$ for $i \in I$ then $\prod_{i \in I_{i}} \mid=\varphi$. (iii) a faithful dependency (formula) if $M_{i} \neq \varphi$ for $i \in$ I iff $\prod_{i \in I_{i}} \models \varphi$. (iv) a limit dependency (formula) if $M_{i} \neq \varphi$ for $i \in I$ then $\lim _{i \in I} M_{i} \mid \varphi$. Here $\lim _{i \in I} M_{i}$ denotes the direct limit of the directed system $M_{i}$, $i \in I$, with respect

to some partial. order on I such that for any two structures $M_{i}, M_{j}$, $i$ and $j$ in $I$, there is a $k$ above both $i, j$ in I such that $M_{i}, M_{j}$ ere mapped in $M_{k}$.

If $N=\prod_{i \in I} M_{j}$. we call the $M_{i}$ 's the factors of $N$. We call a dependency (formula) a

(v) a factor-dependency (formula) if $\prod_{i \in I} M_{i} \neq \varphi$ then $M_{i}=\varphi$ for each $i \in I$.

(vi) a chain-dependency (formula) if $M_{i}$, $i \in I$ is a substructure chain and for each $i \in I M_{i}=0$ then $\underset{i \in I}{U M_{i}}=0$.

Now we can extend Fagin's observation on preservation properties of various dependencies $[F 80]$ :

Proposition 2: (i) The EIDs are product-, chain and limit dependencies.

(ii) The typed EIDs are in addition also factor dependencies, hence faithful.

(iii) The FIDs are also substructure dependencies.

Proof: Consult [CK, exercices to chapter 5].

The model theorist's interest, having its sources in research done in the 1950 s, is in converse theorems, giving rise to syntactical characterizations of various semantically defined classes of formulas.E.g. the substructure formulas are exactly the formulas logically equivalent to universal formulas (i,e. formulas in prenex form with only universal quantifiers). The best source for this in general is[ck, chapter 5$]$ 
With a bit more work we even get a converse:

Theorem 3: Let $\varphi$ be a first order formula true in the trivial structure. Then the following are equivalent:

(i) $\varphi$ is logically to a FID.

(ii) $\varphi$ is both a product and a substructure dependency.

Outline of Proof: (i) $\Rightarrow$ (ii) follows since FIDs are universal Horn-sentences, hence by [CK] both factor and substructure dependencies. To prove (ii) $\Rightarrow$ (i) we note first that $\varphi$ is equivalent to some universal Horn-sentence $\psi$ by [CK] and by proposition $1 \psi$ is equivalent to $\psi \mathrm{F}$. Now, it is easy to see that if $\psi$ is a universal Hom-sentence, so $\psi F$ is equivalent to an FID. In a way this is again "folk-theorem" in the sense of [Ha].

Theorem 4: Let $\varphi$ be a first order formula true in the trivial structure. Then the following are equivalent:

(i) $\varphi$ is logically equivalent to an EID.

(ii) $\varphi$ is both a product and limit dependency.

Outline of Proof: Note first [CK, exercise 5.2.24] that every limit formula is of the form $\hat{\hat{i}}\left(\forall \bar{x}\left(\psi_{i} \rightarrow \exists \bar{y} \theta_{i}\right)\right)$ with $\dot{\psi}_{i}, \theta_{i}$ quantifier free and positive. Now we use the product property to eliminate disjunctions in $\theta_{i}$ and propositional logic to eliminate them in $\psi_{i}$. Then we apply proposition 1 .

\subsection{Critique}

We were rather casual about the proofs of Theorems 3 and 4 because we think that they miss the point. Fagin proved that the (typed) EIDs are faithful dependencies in order to show that they always admit Armstrong relations. We shall show that no such converse theorem can hold in a later section. But our main point here is that algebraic operations alone is not what is needed in data base theory, faithfulness is not a priori interesting and in fact products should be avoided in data base constructions, even for the construction of Armstrong relations.

What we would like are analogues to Theorems 3 and 4 where the algebraic properties are replaced by more practice oriented properties steming from experience with data 
base dependencies. What we have singled out as desirable properties will be discussed in detail in the next two sections.

\section{SECURABILITY}

The following concept has been studied in a different context in [Th, M]. A first order formula $\varphi$ is called n-securable, for $n$ a natural number, if for every structure $M$ (for $\varphi$ ) there is substructure $M_{0}$ of cardinality less or equal to $n$ such that for every structure $M_{1}$ with $M_{0} \subset M_{1} \subset M \quad M_{1} \models \varphi$ iff $M F \varphi$. A formula is securable if we replace less than $n$ by "finite". Let Sec be the set of securable formulae.

The following is immediate from the definition:

Lemma 5: Sec is closed under Boolean operations.

Lemma 6: (i) $F$ and $F_{f}$ coincide on Sec.

(ii) The consequence relation is decidable on Sec (provided we know which formulae are in Sec.

This leaves us with the problem of identifying a securable formula.

Lemma 7: (i) The FIDs are in Sec.

(ii) There is an EID which is not in Sec.

Proof: (i) If an FID is true in $M$ then it is secured by the trivial substructure, otherwise its negation is secured by finitely many witnesses. (ii) By a result in [CLM] the consequence relation on EID is undecidable, contradicting Lemma 6 , if we assume EID is in Sec.

In [Th and $M$ ] the following is proved:

Theorem 8: For a first order formula the following are equivalent:

(i) $\varphi$ is in Sec.

(ii) $\varphi$ is n-securable for some $n$.

(iii) $\varphi$ is equivalent to a Boolean combination of universal sentences.

(iv) $\varphi$ and $-\varphi$ are both chain sentences.

(iii) gives us a syntactic characterization of the securable sentences. Securability 
is stronger than just the finite model property: it is in a sense a continuous finite model property. We claim that securability captures the finite model property inherent in data base theory.

By a classical theorem of model theory, due to Galvin [Ga], [CK], every universal sentence is a Boolean combination of universal Horn-sentences. If we combine this with proposition 1 we get easily

Theorem 9: A dependency $\varphi$ is securable iff it is logically equivalent to a Boolean combination of FIDs.

Outline of Proof: By Theorem $8 \quad \varphi$ is equivalent to a Boolean combination of universal sentences, hence by Galvin's theorem to a Boolean combination of universal Horn-sentences. Now a sentence of the form $\forall \bar{x}\left(\hat{i} A_{i} \rightarrow \underline{F a l s e}\right)$ is equivalent to False by our assumption on the existence of trivial substructures, hence the result.

\section{ARMSTRONG RELATIONS}

Let $S$ be a set of first order formulae, We say that a structure $M$ is an Armstrong-relation for $\Sigma$ a subset of $S$, if $M \neq \Sigma$ but for each $a \in S$ with $\alpha$ not a consequence of $\Sigma, M$ does not satisfy $\alpha$. We say that $S$ is Armstrong if every subset of $\mathrm{S}$, which has a mode1, has an Armstrong-relation. The aim of this section is to study sets of formulae or dependencies which are Armstrong. This approach seems to be new, though it is implicite in [F80].

The following are immediate from the definitions:

Lemma 10: (i) If $S$ is Armstrong and $S_{0}$ is a subset of $S$, then $S_{0}$ is Armstrong.

(ii) If $S$ is Armstrong and $S_{1}$ is such that every $\alpha$ in $S_{1}$ is equivalent to a finite (or infinite) conjunction of elements of $s$, so $\mathrm{S}_{1}$ is Armstrong.

(iii) If $S$ is a set of pairwise inconsistent formulae so $S$ is Armstrong. Note that there are very complex $S$ which are Armstrong, due to (iii). This substantiates our clain that Armstrong alone does not characterize any $S$. In [F80] Fagin proved the following: 
Theorem 11: For a set of first order formulae $s$ the following are equivalent:

(i) $\mathrm{S}$ is Armstrong,

(ii) Whenever $S_{0}, S_{1}$ are subsets of $S$ and $\wedge S_{0}=v S_{1}$ then there is $\alpha$ in $S_{1}$ such that $\wedge S_{0} \vDash \alpha$.

(iii) There is an operation a that maps indexed families $M_{i}$ of models into models $0 M_{i}$ such that for every $\alpha$ in $S \quad \sigma M_{i} \mid=\alpha$ iff for every i $M_{i} \neq \alpha$. (We say that $S$ is 0 -faithful.)

The existence of Armstrong relations seems to be quite fundamental to Data base theory, so it is natural to ask for the class of dependencies to be Armstrong. Fagin proved Theorem 11 for this purpose for it follows that the typed EIDs are Armstrong since they are faithful by proposition 2. But lemma 10 (iii) shows very clearly

that there is no hope to improve Theorem 11 so that Armstrong implies faithfulness. We end this section with a crucial lemma:

Lemma 12: Let $S$ be a set of first oxder formulae and $S_{0}$ be a subset. Let us assume that $S$ is Armstrong and every $\alpha$ in $S$ is equivalent to a Boolean combination of formulae of $S_{0^{\circ}}$. Then $\alpha$ is equivalent to a finite conjunction of formulae in $s_{0}$.

Proof: We first observe that two structures $M_{0}, M_{1}$ satisfy the same sentences of $S$ iff they satisfy the same sentences from $S_{0^{\circ}}$ Now suppose $\alpha$ is in $S$ and is neither valid nor inconsistent. We claim that there is an $\alpha_{0}$ in $S_{0}$ which is not valid and $\alpha \neq \alpha_{0}$.

By Theorem 11 (ii) it is enough to show that:

$\alpha=v\left\{\beta \in S_{0} \mid \beta\right.$ not valid\}. Call this set $S_{1}$. Since $S$ is Armstrong, if this is not the case, there are Armstrong relations $M_{0}, M_{1}$ where all the members of $S_{1}$ are false but $M_{0} F \alpha, M_{1} F-\alpha$, which contradicts the fact that $M_{0}$ and $M_{1}$ satisfy the same sentences from $S_{0}$. Now let $\Sigma$ be $\left\{\beta \in S_{0}|\alpha| \beta\right\}$. Again using that $S$ is Armstrong we conclude that $\alpha$ is equivalent to the conjunction $\Sigma$. But now using compactness we conclude that there is a finite subset $\Sigma_{0}$ of $\Sigma$ such that $\alpha$ is equivalent to the conjunction of $\Sigma_{0}$ Q.E.D. 
5. THE CHARACTERIZATION THEOREM FOR FIDS

Note that by our definition the FIDs are closed under finite conjunctions. So our main theorem reads:

Theorem 13: Let $S$ be a set of dependencies such that:

(i) Each FID is in $\mathrm{S}$

(ii) Each dependency in $S$ is securable

(iii) $\mathrm{S}$ is Armstrong.

Then $S$ consists, up to logical equivalence, exactly of the FIDs.

Proof: This is now a direct consequence of Theorem 9 and Lemma 12.

Note that the EIDs satisfy (i) and (iii) and the class of Boolean combinations of FIDs satisfies (i) and (ii); so theorem 13 is best possible.

If we define that a dependency $\varphi$ is negatively securable if for every $M$ which does not satisfy $\varphi$ there is a finite $M_{0}$ such that for every $M_{1} M_{0} \subset M_{1}=M$ $M_{1}$ does not satisfy $\varphi$; then we can have an analogue theorem for EIDs.

Theorem 14: Let $S$ be a set of dependencies such that:

(i) each EID is in $S$

(ii) each dependency in $S$ is negatively securable

(iii) $\mathrm{S}$ is Armstrong

(iv) each model a subset of $S$ has a trivial submodel.

Then $S$ consists, up to logical equivalence, exactly of the EIDs.

The significance of Theorem 13 is not in possible applications but in its limitative character: It seems to explain why the FIDs are a good class of dependencies and why it cannot be expanded without giving up some of its nice properties. We are fully aware that securability is a rather strong property. It would be interesting to know if we could replace it by a weaker hypothesis such as (a) the consequence relation on $S$ is decidable or (b) each finite subset of $S$ which has a model has a finite model? But it is obvious that then we have to assume stronger closure properties on $S$, such as closure under subformulas. Since these properties will, by Theorem 13, imply securability, Theorem 13 is probably the neatest such characterization. 
Theorem 14 shows how we can relax the securability condition.

\section{UNDECIDABILITY OF SEMANTIC PROPERTIES}

We often are in a situation that we have a set $\Sigma$ of first order formulas which is recursively enumerable and such that every formula having some property $P$ is equivalent to a formula in $\Sigma$. This is the case in theorem $8,9,13,14$. In many cases(here theorem $8,9,13)$ is such that the set of valid sentences in $\Sigma$, call it $\operatorname{val}(\Sigma)$, is recursive. In our context this is mostly so because of the finite model property of the sets in question. It is now natural to ask, whether to have property $P$ is decidable for first order formulas. Vardi observed the following nice theorem:

Theorem 15: (Vardi [Va 2]) Let $\Sigma$ be a set of first order formulas such that $\Sigma$ is recursively enumerable and $\operatorname{Va}(\Sigma)$ is not empty and recursive. Then the set $\mathrm{Eq}(\Sigma)=\{\theta$ : there is a $\sigma \in \Sigma$ such that $\mid=\sigma \leftrightarrow \theta\}$ is not recursive.

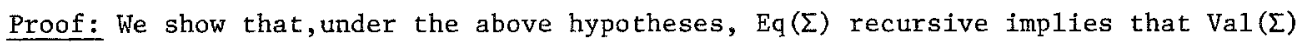
is not recursive. Let $\varphi$ be any sentence. If $\varphi$ is not in $\operatorname{Eq}(\Sigma) \varphi$ is not valid, since $\operatorname{Val}(\Sigma)$ is not empty. But if $\varphi$ is in $E q(\Sigma)$ we can find $\theta$ in $\Sigma$ such that $\varphi$ and $\theta$ are logically equivalent since Eq( $(\Sigma)$ is recursive by our assumption. But then $\varphi$ is valid iff $\theta$ is in $\operatorname{Val}(\Sigma)$. Now, if we assume $\operatorname{Val}(\Sigma)$ recursive, this would give us a decisionprocedure for all valid formulas, which contradicts Godel's theorem.

The following corollary is immediate from theorem $8,9,13$ :

Corollary 16: The following are not recursive:

(i) Eq (FID)

(ii) $\underline{\mathrm{Sec}}$

(iii) For any recursive subset $\Sigma_{0}$ of FIDs or $\underline{\operatorname{Sec}}$ the set $\mathrm{Eq}\left(\Sigma_{0}\right)$.

\section{ACKNOWLEDGEMENT}

We wish to thank C. Beeri, D. Lehmann, M. Makkai and J. Stavi for valuable discussions on the subject. We thank M.Vardi, who suggested section 6 and prevented a stupid error. 
[ABU] Aho, A.V., Beeri, C. and U1lman, J.D.: The theory of joins in relational databases, 19th FOCS (1977), pp. 107-113.

[BV] Beeri, C. and Vardi, M.Y.: On the properties of total join dependencies, Proc. XP1, Workshop on Relationa1 Data Base Theory, Stoney Brook, N.Y. (June 1980).

[Co] Cooper, E.C.: On the expressive power of query languages for relational databases, TR-14-80, Center for Research in Computing Technology, Harvard University (1980).

[CLM] Chandra, A.K., Lewis, H.R. and Makowsky, J.A.: Embedded implicational dependencies and their inference problem, Proc. XP1, Workshop on Relational Data Base Theory, Stoney Brook, N.Y. (June 1980), SIGACT 1981.

[CK] Chang, C.C. and Keisler, H.J.: Model Theory, Studies in Logic and Foundations of Math., Vol. 73 (1973).

[D] Delobel, C.: Normalization and hierarchical dependencies in the relational data mode1, ACM Trans. on Database Systems 3, 3 (1978).

[F77] Fagin, R.: Multivalued dependencies and a new normal form for relational data bases, ACM Trans, on Database Systems 2, 3 (1977).

[F80] Fagin, R.: Horn clauses and database dependencies, ACM Symp. on Theory of Comp., Los Angeles, CA (Apri1 1980), pp. 123-134.

[Ga] Galvin, F.: Horn sentences, Ann. Math. Logic 1, (1970), pp. 389-4-22.

[GJ] Grant, J. and Jacobs, B.E.: On generalized dependencies, to appear.

[Ha] Harel, D.: on Folk theorems, Comm. ACM, July 1980.

[Ke] Keisler, H.J.: Some applications of infinitely long formulas, J. Symbolic Logic 30, (1965), pp. 339-349.

[M ] Makowky, J.A.: Securable quantifiers, k-unions and admissible sets, Logic Co.1loquium 73 (1975), pp. 409-428.

[N] Nicolas, J.M. : First order logic formalization for functional, multivalued and mutual dependencies, ACM-SIGMOD Conf. (1978), pp. 40-46.

[P] Paredaens, J.: Transitive dependencies in a data base scheme, R 387 MBLE, Brussels (1979).

[PP] Parker, D.S., and Parsaye-Ghomi, K.: Inference involving EMVDs and transitive dependencies, TR UCLA.

[PY] Papadimitriou, C. and Yannakakis, M.: Algebraic dependencies, 21st FoCS, Syracuse, N.Y. (October 1980), pp. 328-332.

[R78] Rissanen, J.: Theory of relations for databases - a tutorial survey 7 th Symp. MFCS, Lecture Notes on Comp. Sc. 64 (1978), pp. 536-551.

[SU] Sadri, P. and U1lman, J.D.: A complete axiomatization for a large class of dependencies in relational databases, 12th SIGACT (Apri1 1980).

[SW] Sagiv, Y. and Walecka, S.: Subset dependencies as an al ternative to embedded multivalued dependencies, TR UIUCDCS-R-79-980, Dept. CS, University of Illinois (1979).

[TKY] Tanaka, K.Y., Kambayashi, Y. and Yajima, S.: Properties of embedded muItivalued dependencies in a relational database, TR ER78-03, Dept. of Inf. Sc., University of Kyoto (1978).

[U] U11man, J.D., Principles of Database Systems, Computer Science Press (1980).

[Th] Tharp, L.: Continuity and elementary logic, JSL 39 (1974), pp. 700-716.

[Va] Vardi, M.: The decision problem of database dependencies, preprint June 1980, Wei zmann Institute, Israel.

[Val] Vardi,M. This volume

[Va2] Vardi,M. Oral communication 